\title{
Actividades profesionales confiables en cirugía mínimamente invasiva: iniciando el camino para lograr competencias en el futuro graduado de medicina
}

\author{
Gonzalo Moros I. ${ }^{1}$, Luis Carlos Domínguez T. ${ }^{1}$, Diego Sierra B. ${ }^{1}$, \\ Angélica Villarraga N. ${ }^{2}$ y Juan José Pepín R. ${ }^{1}$
}

Entrustable professional activities in minimally invasive surgery: starting the way to achieve competencies in the future medical graduate

\begin{abstract}
Aims: The development of entrustable professional activities (EPAs) in minimally invasive surgery (MIS) for undergraduates is a need in Colombia. The available evidence is limited. This study aims to describe the preliminary experience with an intervention oriented to this need, embedded in the framework of a competence-based education model (CBE). Materials and Methods: An intervention was designed for the development of EPAs in MIS oriented to the development of attitudes, knowledge and practical skills in medical students. Intervention was delivered through an extended inverted classroom approach. Pre- and postintervention measures were performed by using the Team-STEPPS questionnaire (attitudes), knowledge assessments and OSATS (practical skills). Comparisons were performed by t-test tests $(\mathrm{p}<0.05)$ and the effect size of the intervention was calculated by the Cohen $d$ test. Finally, the student's satisfaction was evaluated. Results: A total of 99 students participated in the study. The intervention showed statistically significant differences $(\mathrm{p}<0.05)$, and great effect on attitudes, knowledge and practical skills $(\mathrm{d}>0.80)$. Likewise, high student satisfaction was evidenced. Discussion: Interactive instructional design fosters development of EPAs in MIS for medical undergraduates. These results are similar to those reported in other interventions under the CBE model.Conclusion: Our intervention showed positive effects on competences oriented to the development of EPAs in MIS for the future graduate. Still is necessary to assess these competencies in real practice and at the end of medical career, in order to evaluate if these activities can be totally reliable to the participants in their future professional practice.
\end{abstract}

Key words: EPA; minimally invasive surgery; competence based-education: practice abilities; simulation.

\section{Resumen}

Objetivos: El desarrollo de actividades profesionales confiables (APROC) para el graduado de medicina en Cirugía Mínimamente Invasiva (CMI) es una necesidad en Colombia. La evidencia disponible es limitada. Este estudio describe la experiencia preliminar con una intervención orientada a esta necesidad, en el marco de un modelo de educación basada en competencias (EBC). Materiales y Métodos: Se diseñó una intervención orientada al desarrollo de actitudes, conocimientos y habilidades prácticas en CMI para estudiantes de medicina, mediante un enfoque de aula invertida extendida. Se realizaron evaluaciones pre y posintervención mediante el cuestionario Team-STEPPS (actitudes), exámenes de conocimiento y OSATS (habilidades prácticas). Se realizaron comparaciones pre y posintervención ( $\mathrm{t}$-test $(\mathrm{p}<0,05)$ y mediciones del tamaño del efecto de la intervención (prueba d Cohen). Finalmente se evaluó la satisfacción estudiantil. Resultados: Un total de 99 estudiantes participaron en el estudio. Se encontraron diferencias estadísticamente significativas $(\mathrm{p}<0,05)$ entre las mediciones pre y posintervención, y gran efecto en las actitudes, conocimientos y habilidades prácticas $(\mathrm{d}>0,80)$. Se evidenció alta satisfacción estudiantil. Discusión: El diseño instruccional a través de metodologías interactivas permite desarrollar APROC en CMI, desde el 
pregrado. Estos resultados son similares a los reportados en otras intervenciones en el marco de la EBC. Conclusión: Nuestra intervención demostró efectos positivos sobre competencias estudiantiles orientadas al desarrollo de APROC en CMI para el futuro graduado. Aun es necesario medir estas competencias en la práctica real y al finalizar la carrera, para determinar si estas actividades pueden ser totalmente confiables a los participantes en su futura práctica profesional.

Palabras clave: APROC; cirugía mínimamente invasiva; educación basada en competencias; habilidades prácticas; simulación.

\section{Introducción}

La cirugía mínimamente invasiva (CMI) ha traído cambios significativos al sistema sanitario y educativo ${ }^{1,2}$. Por una parte, existe la necesidad de afrontar los cambios resultantes en la demanda de servicios y fortalecer los equipos de trabajo. Por otra, existen retos en materia de entrenamiento para cumplir con estas expectativas. No obstante, en economías en desarrollo, ambos sistemas parecen estar desalineados en estos propósitos, o al menos la velocidad a la que avanzan es diferente ${ }^{3}$. Colombia no es una excepción. Por ejemplo, durante los últimos años existe un crecimiento importante en el número y complejidad de CMI en el país, y la participación del médico general como ayudante en equipos de trabajo de cirugía general se ha convertido en una actividad profesional corriente ${ }^{4}$. No obstante, las competencias teóricas, prácticas y actitudinales del médico general para asistir en estos procedimientos requieren todavía desarrollo y esfuerzos por parte del sistema educativo ${ }^{4}$. En el marco de la educación basada en competencias (EBC) $)^{5}$, este reto plantea qué tipo de actividades profesionales en CMI deberían ser confiables al médico general en su práctica diaria, con el fin de promover una práctica segura para el paciente y socialmente responsable $e^{4}$. Estas actividades se conocen como actividades profesionales confiables (APROC) (denominadas Entrustable Professional Activities (EPA) en la literatura anglosajona) $)^{6,7}$. Las APROC del graduado se garantizan una vez este haya adquirido las competencias necesarias durante la carrera, residencia y/o educación continua.

Frente a la necesidad de orientar la educación quirúrgica hacia las APROC en CMI, el Departamento de Cirugía de la Universidad de la Sabana (Colombia) inició un proceso de construcción de un modelo de aprendizaje en CMI (MACMI) para toda la vida ${ }^{4}$, sustentado en los principios del aprendizaje del adulto (experiencial, situado y en contexto) . $^{8}$ Este modelo inicia con el desarrollo de APROC en
CMI para el médico general, mediante la adquisición de competencias específicas en el pregrado, y termina con la educación profesional continuada del cirujano especialista. Para el nivel del médico general se definieron dos APROC: "valorar pacientes en el periodo perioperatorio de procedimientos laparoscópicos básicos" (APROC-1) y "ayudar en procedimientos laparoscópicos básicos" (APROC-2) ${ }^{4}$. Estas APROC se sustentan en once competencias actitudinales, teóricas y prácticas ${ }^{4}$. El presente estudio tiene como objetivo presentar la experiencia preliminar de una intervención dirigida a estudiantes de medicina para el desarrollo de estas APROC, en el marco del modelo de aprendizaje definido.

\section{Materiales y Métodos}

\section{Diseño del estudio y participantes}

Este es un estudio preexperimental pre y posintervención, diseñado para medir el efecto de un curso para el desarrollo de APROC en CMI a nivel del médico general, sobre los conocimientos (fundamentos teóricos), habilidades (transferencia, profundidad, corte y precisión) y actitudes estudiantiles (liderazgo, proactividad, comunicación y colaboración). Se invitaron a participar voluntariamente a 100 estudiantes de cuarto año de medicina durante la asignatura de cirugía general (Universidad de la Sabana, Colombia). El curso se llevó a cabo durante los meses de agosto y septiembre de 2017. El estudio fue aprobado por la Comisión de Educación Médica (Facultad de Medicina, Universidad de la Sabana).

\section{Intervención}

El curso fue desarrollado en coherencia y alineación con las APROC 1 y 2 del MACMI, en su primer nivel de entrenamiento (médico general). El diseño curricular fue realizado entre octubre de 2016 y junio de 2017, bajo el enfoque de tareas globales de 4C/ID (cuatro componentes/diseño ins- 
truccional), que especifica las tareas de aprendizaje, información de soporte, información procedimental y tareas prácticas ${ }^{9}$. Metodológicamente, el currículo fue entregado a los estudiantes a través de una aula invertida extendida (AIE). El AIE se define como "un modelo interactivo de enseñanza/aprendizaje que involucra no solo las actividades fuera del aula y las actividades convencionales en clase del enfoque clásico del aula invertida, sino que también incorpora o "extiende el aula" hasta los escenarios controlados en el laboratorio de simulación"10. En otras palabras el AIE pretende entregar al estudiante los materiales de una clase, para su estudio independiente, alojados en una plataforma virtual (actividades "fuera del aula"). Luego, el espacio tradicional de la clase se emplea para la enseñanza interactiva, comúnmente mediante la discusión de casos, y se extiende hasta el laboratorio de simulación para el desarrollo de habilidades prácticas (actividades "en el aula"). El AIE se desarrolló en cuatro fases.

La primera fase consistió en actividades de autoaprendizaje "fuera del aula" (dos semanas), mediante la revisión de videos y documentos previamente diseñados por los investigadores teniendo en cuenta las competencias en CMI para el médico general: 1) liderazgo, comunicación, proactividad y colaboración; 2) alcance y limitaciones de la CMI; 3) funcionamiento de la torre de laparoscopia; 4) instrumental laparoscópico básico; 5) ubicación de recursos técnicos en la sala de cirugía; 6) posición del paciente; 7) lista de chequeo en cirugía; 8) uso de la cámara de laparoscopia; 9) complicaciones de la cirugía laparoscópica; 10) exposición del campo operatorio y 11) principios básicos del cierre de heridas quirúrgicas. También se diseñó la información procedimental y guías prácticas de simulación para ejercicios de transferencia, profundidad, corte y precisión en cirugía laparoscópica. La segunda fase consistió en el desarrollo de actividades "en el aula", las cuales se extendieron al laboratorio de simulación (12 h). Durante esta fase, las tareas prácticas fueron desarrolladas en cuatro estaciones de trabajo: 1) liderazgo, comunicación, proactividad y colaboración en CMI enfocadas a la seguridad del paciente (discusiones de caso); 2) funcionamiento y posición de la torre de laparoscopia - funcionamiento de la cámara de laparoscopia (laboratorio de simulación); 3) instrumental y ejercicios básicos de transferencia, profundidad, corte y precisión en simulador de mediana fidelidad (pelvi-trainer) $\mathrm{y}$; 4) principios de anudado y sutura básica en especímenes (laboratorio de simulación). La tercera fase consistió en práctica independiente de habilidades en simulador de mediana fidelidad. Para esta fase, se estableció que los estudiantes debían completar al menos cinco horas de trabajo autodirigido en un período de dos semanas. Finalmente, la cuarta fase se focalizó en retroalimentación y evaluación de calidad y satisfacción del estudiante con el curso.

\section{Desenlaces, instrumentos y recolección de información}

El desenlace primario fue el impacto del AIE en CMI en las actitudes, conocimiento y habilidades prácticas del estudiante. Las actitudes fueron evaluadas mediante el cuestionario Team-STEPPS diseñado por la Agency for Healthcare Research and Quality (AHRQ) (https://www.ahrq.gov). El cuestionario consta de 30 preguntas calificadas mediante una escala de Likert ( 1 = fuertemente en desacuerdo; 5 = fuertemente de acuerdo), distribuidas en cinco subescalas (liderazgo, monitoreo situacional, soporte mutuo, comunicación y estructura de equipo de trabajo). La consistencia interna de las subescalas (Cronbach $\alpha$ ) varía de 0,70 (estructura del equipo) a 0,83 (monitoreo situacional) ${ }^{11}$. Los conocimientos teóricos fueron evaluados mediante pruebas de selección múltiple (15 preguntas), y las habilidades prácticas mediante evaluación objetiva estructurada de habilidades técnicas (conocidas en la literatura anglosajona como objective structured assessment of technical skill -OSATS-) ${ }^{12}$ de acuerdo a listas de chequeo diseñadas por los investigadores para cada una de las cuatro habilidades prácticas (rango 0 a 5). Todas las evaluaciones fueron de carácter formativo. Finalmente, la satisfacción estudiantil se evaluó mediante un cuestionario institucional que determina la pertinencia, coherencia y contribución de la actividad a la formación del estudiante. El cuestionario consta de 5 preguntas calificadas mediante una Escala de Likert $(1=$ malo; $5=$ excelente $)$.

El Team-STEPPS y los conocimientos teóricos fueron medidos dos semanas previas al inicio del curso (antes de las actividades "fuera del aula"), y las habilidades prácticas fueron evaluadas al inicio de la segunda fase. Posteriormente, tras la intervención, se evaluaron nuevamente las actitudes, conocimientos y habilidades prácticas, dos semanas después de finalizar la tercera fase de la intervención (luego de la práctica independiente de habilidades en simulador de mediana fidelidad). Para este propósito fue diseñado un nuevo examen de conocimiento teórico de 15 preguntas de complejidad similar al inicial. Al finalizar el curso se realizó una medición de la satisfacción estudiantil. La recolección de toda la información fue realizada digitalmente, mediante cuestionarios administrados de forma anónima, voluntaria y confidencial. 


\section{Análisis estadístico}

Inicialmente se calcularon las medias, desviaciones estándar (DE) y rangos del Team-STEPPS y sus subescalas, pruebas teóricas y evaluaciones prácticas para cada uno de los dos momentos de medición. La consistencia interna del Team-STEPPS se calculó mediante el coeficiente alfa de Cronbach (adecuado si $>0,70)^{13}$. Luego, se realizaron comparaciones entre los resultados pre y posintervención mediante la prueba de $t$-student (significativa si $p<0,05$ ) y el cálculo del tamaño del efecto. Para este último, se utilizó la prueba $d$ de Cohen para cada subescala y puntaje global del Team-STEPPS, pruebas teóricas y evaluación práctica (intervalo de confianza $95 \%$ (IC 95\%). Los criterios de referencia para interpretar el tamaño del efecto fueron: efecto pequeño $d= \pm 0,20$, efecto medio $d= \pm 0,50$ y efecto grande $d= \pm 0,80^{14}$. Finalmente se calcularon las estadísticas descriptivas para los ítems relacionados con las medidas de satisfacción estudiantil. El análisis se realizó en la versión-14 del programa Stata (Stata Corp. USA).

\section{Resultados}

Un total de 99 estudiantes participaron voluntariamente en el estudio (99\%). La edad promedio fue $21,7 \pm 1,35$ años (20-27) (69,23\% del género femenino). El porcentaje de respuesta en las evaluaciones preintervención fue: conocimientos $(100 \%)$, habilidades $(98,9 \%)$ y actitudes $(76,7 \%)$. El porcentaje de respuesta en las evaluaciones posintervención fue: conocimientos $(88,8 \%)$, habilidades $(100 \%)$ y actitudes $(88,8 \%)$. El $97,9 \%$ de los participantes completó la evaluación de satisfacción. Los coeficientes de confiabilidad (alfa de Cronbach) para el Team-STEPPS fueron: 0,86 (preintervención) y 0,82 (posintervención).

\section{Comparaciones pre y posintervención:} actitudes, conocimientos y habilidades prácticas

a. Actitudes: La media, DE y rango para el TeamSTEPPS fue: $3,73 \pm 0,5(2,82-4,0)$ (preintervención) versus $4,23 \pm .24(3,52-4,86)$ (posintervención) $(\mathrm{p}<0,05)$. El tamaño del efecto fue $\mathrm{d}=1,97$ (IC95\% 1,59-2,34).

b. Conocimientos: Los resultados de la evaluación fueron: 2,58 $\pm 0,62(0,3-5,0)$ (preintervención) versus 3,48 $\pm 0,54(2,3-4,6)$ (posintervención) $(\mathrm{p}<0,05)$. El tamaño del efecto fue $\mathrm{d}=1,52$ (IC 95\% 1,20-1,85).

c. Habilidades prácticas: Los resultados globales de la evaluación fueron: $3,43 \pm 0,85(0-5,0)$ (prein- tervención) versus $4,75 \pm 0,39(3,0-5,0)$ (posintervención) $(\mathrm{p}<0,05)$. El tamaño del efecto fue $d=2,0$ (IC 95\% 1,65-2,34). El tiempo promedio de práctica independiente de los estudiantes fue: $4,24 \mathrm{~h} \pm 2,86$ (2-19).

Las estadísticas descriptivas y el tamaño del efecto discriminados para las subescalas del TeamSTEPPS, evaluación de conocimientos y habilidades prácticas se encuentran en la Tabla 1.

\section{Satisfacción estudiantil}

Los resultados globales de satisfacción estudiantil con la actividad fueron: 4,32 $\pm 0,67(2,0-5,0)$. Todos los ítems de la satisfacción en cuanto a su pertinencia, coherencia y contribución a la formación integral fueron superiores a 4,0 (Tabla 2).

\section{Discusión}

Los hallazgos del presente estudio indican que el entrenamiento en CMI, dirigido al primer nivel del modelo de aprendizaje experiencial en CMI (MAC$\mathrm{MI}$ ), tuvo un impacto positivo (magnitud grande del efecto) en los conocimientos básicos, habilidades prácticas y actitudes de estudiantes de medicina. Igualmente se evidenció una alta satisfacción.

Estos hallazgos se explican por varias razones. En primer lugar, por el diseño instruccional que integra intervenciones simultáneas en los tres dominios de competencia (conocimientos, habilidades y actitudes), a través de metodologías de enseñanza/ aprendizaje interactivas como el AIE. Evidencia preliminar soporta la relación positiva de este tipo de intervenciones con el aprendizaje autodirigido, interés y motivación estudiantil ${ }^{9,15,16}$. En segundo lugar, porque nuestro modelo se sustenta en los principios del aprendizaje del adulto. Este marco teórico tiene en cuenta la relevancia del aprendizaje situado, experiencial y en contexto ${ }^{8}$. A través de experiencias concretas, mediante el uso de simulación de mediano grado de fidelidad, se pretende estimular el interés del estudiante hacia el objeto de conocimiento (CMI) y orientarlo hacia su relevancia para la solución de problemas en contextos reales. Estudios previos respaldan estos beneficios de la simulación clínica en el aprendizaje situado en cirugía ${ }^{17-19}$. Finalmente, creemos que los efectos positivos son explicados por la relevancia observada por los estudiantes para el desarrollo de APROC en cirugía, en el marco del ejercicio profesional del graduado. Permitir que los estudiantes reflexionen sobre la importancia y rol fundamental del médico general en equipos de trabajo, ofrece una oportunidad única 
Tabla 1. Diferencias pre y posintervención en actitudes, conocimientos y habilidades prácticas

\begin{tabular}{|c|c|c|c|c|}
\hline \multicolumn{5}{|l|}{ 1. Actitudes } \\
\hline Actitudes (Team-STEPPS) & Media, DE, rango & Media, DE, rango & $\mathbf{p}$ & $\begin{array}{l}\text { Tamaño del efecto } \\
\text { d Cohen (IC 95\%) }\end{array}$ \\
\hline Liderazgo & $3,89 \pm 0,23(3-4)$ & $4,73 \pm 0,29(4-5)$ & 0,0001 & $3,09(2,63-3,54)$ \\
\hline Monitoreo de situaciones & $3,88 \pm 0,27(2,6-4)$ & $4,59 \pm 0,37(3,17-5)$ & 0,0001 & $2,11(1,72-2,49)$ \\
\hline Soporte mutuo & $3,4 \pm 0,55$ & $3,38 \pm 0,41(2,5-5)$ & 0,6 & $-0,04(-0,35-0,26)$ \\
\hline Global & $3,73 \pm 0,25(2,82-4)$ & $4,23 \pm 0,24(3,52-4,86)$ & 0,0001 & $1,97(1,59-2,34)$ \\
\hline \multicolumn{5}{|l|}{ 2. Conocimientos } \\
\hline Prueba & Media, DE, rango & Media, DE, rango & $\mathbf{p}$ & $\begin{array}{l}\text { Tamaño del efecto } \\
\text { d Cohen (IC 95\%) }\end{array}$ \\
\hline Evaluación teórica de conceptos & $2,58 \pm 0,62(0,3-5)$ & $3,48 \pm 0,54(2,3-4,6)$ & 0,0001 & $1,52(1,20-1,85)$ \\
\hline Transferencia & $3,6 \pm 1,24 \quad(0-5)$ & $4,87 \pm 0,52(2-5)$ & 0,0001 & $1,33(1,02-1,64)$ \\
\hline Profundidad & $3,09 \pm 1,46(0-5)$ & $4,8 \pm 0,61 \quad(1-5)$ & 0,0001 & $1,52(1,21-1,84)$ \\
\hline Corte & $3,07 \pm 1,34(0-5)$ & $4,39 \pm 1,01(1-5)$ & 0,0001 & $1,1 \quad(0,8-1,40)$ \\
\hline Precisión & $3,95 \pm 1,2 \quad(0-5)$ & $4,93 \pm 0,37(2-5)$ & 0,0001 & $1,09(0,79-1,39)$ \\
\hline Global & $3,43 \pm, 85 \quad(0-5)$ & $4,75 \pm 0,39(3-5)$ & 0,0001 & $2 \quad(1,65-2,34)$ \\
\hline
\end{tabular}

DE: Desviación estándar,

Tabla 2. Satisfacción estudiantil con la intervención $(\mathbf{n}=97)$

\begin{tabular}{|lcc|}
\hline Ítem & Media \pm DE (rango) & IC 95\% \\
\hline 1. Temas evaluados/tratados & $4,35 \pm 0,79(2-5)$ & $4,19-4,50$ \\
\hline 2. Coherencia de los objetivos formulados en la actividad & $4,42 \pm 0,73(2-5)$ & $4,27-4,57$ \\
\hline 3. Pertinencia y actualización del tema que se evaluó/trató & $4,42 \pm 0,85(1-5)$ & $4,25-4,59$ \\
\hline 4. Contribución a la formación integral del estudiante/participante & $4,32 \pm 0,92(1-5)$ & $4,14-4,51$ \\
\hline 5. Ejemplos y experiencias reales aprovechadas en el desarrollo de la evaluación & $4,44 \pm 0,79(2-5)$ & $4,28-4,60$ \\
\hline Global & $4,32 \pm 0,67(2-5)$ & $4,19-4,46$ \\
\hline
\end{tabular}

DE: Desviación estándar.

para la educación quirúrgica y la transformación de los esquemas tradicionales de enseñanza.

Este estudio tiene fortalezas y limitaciones. En cuanto a las primeras se encuentra el diseño instruccional (4C/ID) y metodológico (AIE) riguroso, en el marco de un modelo experiencial para la enseñanza a lo largo de la vida ${ }^{9,10}$. Aunque aún se requieren nuevas mediciones, creemos que nuestra propuesta apuesta a la continuidad educativa y alineación del pregrado, postgrado y educación permanente en sus aspectos curriculares. En este contexto una fortaleza de esta intervención es que responde a una construcción coherente y holística sobre la enseñanza de CMI que visualiza en conjunto el futuro desarrollo de APROC para el médico general, residente y cirujano. En otras palabras, busca fortalecer aquellas actividades en CMI que se confiarían a un médico general en la práctica diaria. También es una fortaleza el uso de instrumentos validados (Team-STEPPS) y el desarrollo de OSATS ${ }^{12}$ para 
la medición de habilidades. No obstante, este es un estudio preliminar que involucra un primer paso en el camino de las APROC en CMI para el médico general. Aun es necesario incorporar la medición de competencias en la práctica y efectuar una evaluación global al finalizar la carrera, con el fin de determinar si las actividades en CMI son totalmente confiables a los participantes para que su práctica profesional sea socialmente responsable y segura. Igualmente este estudio fue realizado en una sola institución de carácter privado, lo cual puede limitar la generalización de los resultados. Invitamos a realizar investigaciones en otros contextos.

Este estudio tiene implicaciones para la práctica e investigación. En la práctica permite fortalecer el diseño instruccional enfocado al desarrollo de las APROC para el graduado. Creemos que este enfoque permite ver más allá de cursos y asignaturas aisladas de la realidad profesional. Por otra parte permite alinear la estructura curricular de diversos niveles de entrenamiento bajo los mismos principios conceptuales del aprendizaje experiencial. Finalmente, aporta a la transformación del paradigma de la educación médica tradicional centrado en la alta transmisión de información, memoria y baja interactividad. Al respecto tiene implicaciones para el desarrollo profesoral (faculty development). Las oportunidades de investigación futura, a parte de las mencionadas, tienen que ver con la forma de evaluar las APROC en CMI en el graduado. Esta evaluación deberá enfocarse en la capacidad de retención de los fundamentos en CMI a mediano y largo plazo, curva de aprendizaje, experiencia en contextos reales, certificación y vigencia futura de las APROC. También ofrece oportunidades para evaluar nuestra propuesta de aprendizaje para toda la vida desde la base (médico general) hasta el cirujano especialista.

En conclusión, hemos presentado los resultados exitosos de una intervención, desarrollada a partir de un diseño instruccional experiencial en CMI para el estudiante de medicina, la cual constituye la base de un modelo de aprendizaje para toda la vida. Este modelo tiene implicaciones para la práctica y abre oportunidades de investigación futura.

\section{Responsabilidades éticas}

Protección de personas y animales. Los autores declaran que los procedimientos seguidos se conformaron a las normas éticas del comité de experimentación humana responsable y de acuerdo con la Asociación Médica Mundial y la Declaración de Helsinki.

Confidencialidad de los datos. Los autores declaran que han seguido los protocolos de su centro de trabajo sobre la publicación de datos de pacientes.

Derecho a la privacidad y consentimiento informado. Los autores declaran que en este artículo no aparecen datos de pacientes.

\section{Referencias}

1. Mutter D, Dallemagne B, Perretta S, Vix M, Leroy J, Pessaux P, et al. Innovations in minimally invasive surgery: lessons learned from translational animal models. Langenbecks Arch Surg. 2013;398:91923.

2. de Ruijter V, Halvax P, Dallemagne B, Swanström L, Marescaux J, Perretta S. The Business Engineering Surgical Technologies (BEST) teaching method: incubating talents for surgical innovation. Surg Endosc. 2015;29:48-54.

3. Chao TE, Mandigo M, Opoku-Anane J, Maine R. Systematic review of laparoscopic surgery in low- and middleincome countries: benefits, challenges, and strategies. Surg Endosc. 2016;30:110.

4. Domínguez LC, Moros G.
Responsabilidad Social y Educación Basada en Competencias en Cirugía: ¿Se deben confiar actividades profesionales en Cirugía Mínimamente Invasiva al médico general? Submitted (Revista Chilena de Cirugía).

5. Frank JR, Snell L, Englander R, Holmboe ES, ICBME Collaborators. Implementing competency-based medical education: Moving forward. Med Teach. 2017;39:568-73

6. Ten Cate O, Chen HC, Hoff RG, Peters $\mathrm{H}$, Bok H, van der Schaaf M. Curriculum development for the workplace using Entrustable Professional Activities (EPAs): AMEE Guide No. 99. Med Teach. 2015;37:983-1002.

7. Ten Cate O. Guía sobre las actividades profesionales confiables. FEM 2017;20:95-102.

8. Kolb D, Fry R. Toward an applied theory of experiential learning. in C. Cooper (ed.), Theories of Group Process, London: John Wiley, 1975.

9. van Merriënboer JJG, Kirschner PA. Ten Steps to Complex Learning: A Systematic Approach to Four-Component Instructional Design 2nd Edition Routledge, 2012.

10. Domínguez LC, Sierra D, Pepin JJ, Moros A, Villarraga Al. Efecto del Aula Invertida Extendida a simulación clínica para la resucitación del paciente traumatizado: un estudio piloto evaluando las percepciones estudiantiles sobre el aprendizaje. Rev Colomb Anestesiol. 2017. http://dx.doi.org/10.1016/j. rca.2017.07.011.

11. Sexton JB, Helmreich RL, Neilands TB, Rowan K, Vella K, Boyden J, et al. The safety attitudes questionnaire: Psychometric properties, benchmarking 
ARTíCULO ORIGINAL

data, and emerging research. BMC Health Serv Res. 2006;6:44. Published online 2006 Apr 3. doi: 10.1186/1472-6963-6-44.

12. Hatala R, Cook DA, Brydges R, Hawkins R. Constructing a validity argument for the Objective Structured Assessment of Technical Skills (OSATS): a systematic review of validity evidence. Adv Health Sci Educ Theory Pract. 2015;20:1149-75.

13. Cronbach LJ, Warrington WG. Timelimit tests: estimating their reliability and degree of speeding. Psychometrika 1951;16:167-88.
14. Cohen J. Statistical power analysis for the behavioural sciences (2nd Ed). Hillsdale, NJ: Lawrence Erlbaum Associates, 1998.

15. Chen F, Lui AM, Martinelli SM. A systematic review of the effectiveness of flipped classrooms in medical education. Med Educ. 2017;51:585-97.

16. Rotellar C, Cain J. Research, Perspectives, and Recommendations on Implementing the Flipped Classroom. Am J Pharm Educ. 2016;80(2):34. doi: 10.5688/ajpe80234.

17. Cannon-Bowers JA, Bowers C, Procci $\mathrm{K}$. Optimizing learning in surgical simulations: guidelines from the science of learning and human performance. Surg Clin North Am. 2010;90:583-603.

18. Cleland J, Walker KG, Gale M, Nicol LG. Simulation-based education: understanding the socio-cultural complexity of a surgical training 'boot camp'. Med Educ. 2016;50:829-41.

19. Doumouras AG, Keshet I, Nathens AB, Ahmed N, Hicks CM. A crisis of faith? A review of simulation in teaching teambased, crisis management skills to surgical trainees. J Surg Educ. 2012;69:274-81. 